\title{
Heart diastolic dysfunction in patients with systemic sclerosis
}

\author{
Michał Ciurzyński ${ }^{1}$, Piotr Bienias ${ }^{1}$, Katarzyna Irzyk ${ }^{1}$, Maciej Kostrubiec ${ }^{1}$, Agnieszka Szewczyk², \\ Urszula Demkow ${ }^{3}$, Maria Siwicka², Katarzyna Kurnicka' ${ }^{1}$, Barbara Lichodziejewska ${ }^{1}$, Piotr Pruszczyk ${ }^{1}$
}

\begin{abstract}
${ }^{1}$ Department of Internal Medicine and Cardiology, Medical University of Warsaw, Warsaw, Poland

2Department of Dermatology, Medical University of Warsaw, Warsaw, Poland ${ }^{3}$ Department of Laboratory Diagnostics and Clinical Immunology of Developmental Age, Medical University of Warsaw, Warsaw, Poland
\end{abstract}

Submitted: 6 June 2013

Accepted: 14 October 2013

Arch Med Sci 2014; 10, 3: 445-454

DOI: 10.5114/aoms.2014.43739

Copyright @ 2014 Termedia \& Banach

\section{Abstract}

Introduction: There are limited data on left (LV) and right ventricular (RV) diastolic function in systemic sclerosis (SSc) patients especially in relation to biomarkers of matrix remodeling. The aim of the study was to analyze LV and RV myocardial diastolic function in SSc patients at baseline and after at least 1 year of follow-up and its relation to serum tissue inhibitors of metalloproteinase 1 (TIMP-1) level.

Material and methods: We prospectively studied 111 SSc patients (101 female, 10 male, age $54.2 \pm 13.8$ years) and 21 age-matched controls (18 female, 3 male, age $49.3 \pm 10.5$ years). After at least 1 year of observation (3.0 \pm 1.1 years) we reevaluated 69 of the SSc patients. Transthoracic echocardiography (Philips, iE33) for assessment of LV and RV diastolic function was performed and TIMP-1 serum level was measured.

Results: Impaired LV relaxation was observed in 38 (34\%) SSc patients and in $1(5 \%)$ of the controls $(p<0.001)$. The mean E/A ratio was lower in patients with SSc than in controls $(p=0.002)$ and significantly decreased after the follow-up period $(p=0.02)$. Impaired RV relaxation was detected in $25(22.5 \%)$ SSc patients and in $1(5 \%)$ control subject $(p<0.001)$ but did not deteriorate after follow-up. Mean serum level of TIMP-1 was significantly elevated in the follow-up group compared to baseline examination ( $p=$ $0.0001)$. Serum TIMP-1 level correlated positively with E/E', both septal and lateral $(r=0.4, p=0.002$ and $r=0.32, p=0.01)$.

Conclusions: The LV and RV relaxation is impaired in SSc patients. Moreover, left ventricular diastolic function deteriorated after the follow-up period. The TIMP-1 serum levels correlate with echocardiographic parameters, providing a potent link for LV diastolic function and matrix remodeling in patients with SSc.

Key words: systemic sclerosis, echocardiography, diastolic function, biomarkers.

\section{Introduction}

Systemic sclerosis (SSc) is characterized by increased collagen deposition and widespread vascular lesions leading to progressive systemic fibrosis [1]. In the clinical course of SSc, extensive fibrous degeneration develops in various internal organs, including the heart [2]. It was sug-

\author{
Corresponding author: \\ Michał Ciurzyński MD, PhD \\ Department of Internal \\ Medicine and Cardiology \\ Medical University of Warsaw \\ 4 Lindleya St \\ 02-005 Warsaw, Poland \\ Phone: +48501730654 \\ E-mail: michal.ciurzynski@ \\ wum.edu.pl
}


gested that tissue fibrosis observed in SSc is partially related to matrix metalloproteinases (MMP) inhibition by tissue inhibitors of metalloproteinases (TIMP) [3]. Serum concentrations of MMP-1 was reported to be increased in patients with SSC $[4,5]$. Moreover, serum levels of TIMP-1 were also elevated in SSc patients [4, 6-8].

Such pathogenesis of organ complications in SSc suggests that the impairment of diastolic function of both ventricles might also be present. Diastolic dysfunction is clinically important and according to recent data is a marker of increased risk of death in SSc patients [9]. There are several studies evaluating left (LV) but only a few on right ventricular (RV) diastolic function in SSc patients [10-14]. Moreover, there is limited evidence on potential progression of diastolic dysfunction during follow-up [15].

The aim of our study was to assess LV and RV diastolic function with Doppler echocardiography and to evaluate the potential relationship to serum TIMP-1 concentration in systemic sclerosis patients at baseline and after at least 1 year of follow-up.

\section{Material and methods}

One hundred eleven consecutive patients with confirmed SSc (101 females, 10 males, mean age $54.2 \pm 13.7)$ with mean time of $9.4 \pm 11.4$ years from SSc diagnosis were prospectively included in the study. The SSc was diagnosed according to the American College of Rheumatology criteria [16]. Clinical characterization of patients with SSc was performed according to the European Scleroderma Trial and Research (EUSTAR) recommendations [17]. Both patients with diffuse SSc and limited scleroderma were recruited. Forty-seven (42\%) patients were classified as having diffuse SSc (dSSc), while 64 (58\%) others had limited SSc (ISSc).

Patients with cardiovascular disorders such as coronary artery disease (angina pectoris, previous myocardial infarction, ECG and/or echocardiographic signs of myocardial ischemia), atrial fibrillation, cardiac pacing, significant valvular abnormalities, LV hypertrophy (intraventricular septum and posterior wall $>11 \mathrm{~mm}$ in echocardiographic examination) were not included in the current study. Moreover, SSc patients with significant pulmonary function abnormalities defined by forced vital capacity (FVC), total lung capacity (TLC), or forced expiratory volume in $1 \mathrm{~s}\left(\mathrm{FEV}_{1}\right)<60 \%$ of the predicted value were also not included. Impaired renal function with creatinine clearance calculated by the Modification of Diet in Renal Disease Study Group (MDRD) formula below $60 \mathrm{ml} / \mathrm{min}$ was also an exclusion criterion.

As a control group we assessed 21 age- and sex-matched subjects (18 females, 3 males, mean age $49.3 \pm 10.5$ years). Special care was taken to select the control group with the same profile of coexisting systemic hypertension and treated with the hypotensive therapy as used in the study group. These subjects had no data for pulmonary diseases and presented no echocardiographic evidence of structural heart disease.

After at least 1 year of observation $(3.0 \pm 1.1$ years, median 3.1 years, range: $1.0-4.8$ years) the second evaluation of SSc patients was done.

All patients and control subjects gave informed consent, and the protocol of the study was approved by the local Institutional Ethics Committee.

\section{Clinical features and visceral involvement}

Clinical evaluation for the signs and symptoms of SSc was performed in all patients at the onset of the study. In all SSc patients pulmonary involvement was assessed by chest radiography, high-resolution computed tomography (Toshiba Aquilion 64), pulmonary function testing and measurement of single breath diffusing lung capacity for carbon monoxide (DECO) (Sensor Medics, Yorba Linda, USA).

\section{Serological parameters}

Standard techniques were used to screen all patients for the serological markers anti-nuclear antibody (ANA), anti-nucleolar antibody (ANoA) and anti-centromere antibody (ACA) by indirect immunofluorescence on monkey esophagus at a dilution of serum of $1: 20$ and on the Hep 2 cell line at a dilution of $1: 40$. Anti-extractable nuclear antigen (ENA) antibodies, including Scl-70 antibodies, were identified by double immunodiffusion.

\section{Blood sampling and assays}

Fasting blood samples were collected by venipuncture, centrifuged and sera were stored at $-70^{\circ} \mathrm{C}$ until assayed.

A commercial immunoenzymatic kit to detect human tissue inhibitor of metalloproteinases 1 (TIMP-1), Human TIMP-1 Immunoassay (R\&D Systems, Inc. Minneapolis, MN, USA), was used. The tests detecting TIMP-1 are based on a solid double antibody sandwich ELISA. All samples were assayed in duplicate. In the positive cases, antigen-antibody complex reacted with peroxidase-labeled antihuman IgG conjugate. Using $\mathrm{H}_{2} \mathrm{O}_{2} / \mathrm{TMB}$ as substrate, the enzymatic activity of peroxidase was measured at $450 \mathrm{~nm}$ using the automated reading system Asys UVM 340. All the results were referred to the standard curve. The standards were provided for the generation of a semi-logarithmic reference curve. Immuno-enzymatic assays were performed blindly by a technician according to the manufacturer's instructions. 


\section{Echocardiography}

Echocardiographic examination was performed with a Philips iE33 ultrasound system (Andover, Md., USA) with 2.5-3.5 MHz transducers. Patients were examined in the left lateral position. Measurements were averaged over 5 consecutive heart cycles.

\section{Evaluation of left ventricular systolic function}

Left ventricular ejection fraction (LVEF) was calculated according to Simpson's formula employing a two-dimensional image of the LV chamber during systole and diastole in the four- and two-chamber apical views [18].

\section{Evaluation of right ventricular systolic function}

In order to assess the RV function using the one-dimensional M-mode echocardiography, the tricuspid annulus plane systolic excursion (TAPSE) was measured. We also calculated RV fraction area change (FAC) defined as end diastolic area end systolic area/end diastolic area $\times 100 \%$ [19].

\section{Assessment of right ventricle systolic pressure}

Using continuous-wave Doppler echocardiography, the tricuspid regurgitation peak gradient (TRPG) was calculated according to the simplified Bernoulli equation. Pulmonary hypertension (PH) was suspected according to European Society of Cardiology (ESC) criteria when TRPG > $31 \mathrm{~mm} \mathrm{Hg}$ (tricuspid regurgitant velocity $>2.8 \mathrm{~m} / \mathrm{s}$ ) [20]. Patients with suspicion of $\mathrm{PH}$ were referred for right heart catheterization ( $\mathrm{RHC})$.

\section{Assessment of left and right atrial dimensions}

The dimension of the left atrium (LA) was measured in the parasternal long-axis view in late diastole defined by the R wave of continuously monitored ECG lead. Both upper to lower and medial to lateral dimensions of the left and right atrium were measured on two-dimensional images from apical four-chamber view at the end of systole.

\section{Analysis of left ventricular diastolic performance}

Mitral valve inflow (MVF) was recorded in the apical four-chamber view with the pulsed wave Doppler gate positioned in the LV at the level of the mitral valve edges. The following parameters were measured: peak velocity of the early inflow phase $(E)$, peak velocity of the atrial inflow phase
(A), E/A ratio and phase $\mathrm{E}$ deceleration time (DT). The apical five-chamber view enabled simultaneous registration of the flow pattern through aortic and mitral valves and then isovolumetric relaxation time (IVRT) was calculated. The pattern of the right upper pulmonary vein inflow (PVF) was recorded from the apical four-chamber view after the Doppler gate was positioned approximately $1 \mathrm{~cm}$ inside the vein. The following parameters were evaluated: peak systolic inflow velocity (S), peak diastolic inflow velocity (D), S/D ratio and peak velocity of regurgitated atrial flow (Ar) (Figure 1).

\section{Tissue Doppler mitral annular early diastolic velocities}

Tissue Doppler imaging (DTI) was performed in the apical views to acquire mitral annular velocities. The sample volume was positioned at or $1 \mathrm{~cm}$ within the septal and lateral insertion sites of the mitral leaflets and adjusted as necessary (usually $5-10 \mathrm{~mm}$ ) to cover the longitudinal excursion of the mitral annulus in diastole. Lateral annulus early diastolic velocity (Mit E' lateral) and septal annulus early diastolic velocity (Mit E' septal) were measured. Moreover, mitral E/E' lateral and septal were calculated.

The following values were considered normal: E/A $1.28 \pm 0.25$, DT $181 \pm 19 \mathrm{~ms}$, IVRT $74 \pm 7 \mathrm{~ms}$, S/D $1.21 \pm 0.2$, Ar $23 \pm 3 \mathrm{~cm} / \mathrm{s}$, lateral E' $16.1 \pm 2.3$ $\mathrm{cm} / \mathrm{s}$ and septal E' $12.2 \pm 2.3 \mathrm{~cm} / \mathrm{s}$. Moreover, E/E' ratio > 15 was defined as abnormal [21].

\section{Assessment of global left ventricular function}

The index of LV efficiency (Tei index) as a sum of isovolumetric systole and diastole time to LV ejection time ratio was calculated according to the method proposed by Tei et al. The normal value for the LV was assumed to be $0.39 \pm 0.05$ [22] (Figure 2).
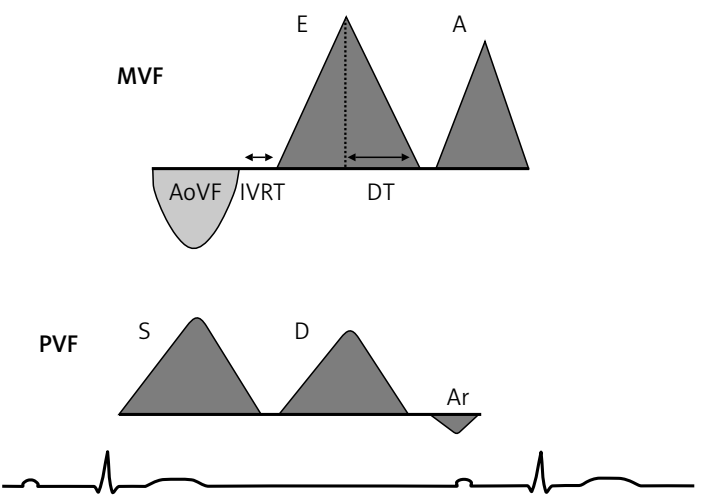

Figure 1. Methods of mitral valve inflow (MVF) and pulmonary vein inflow (PVF) measurements

AoVF - aortic valve flow. Other abbreviations are given in the main text 


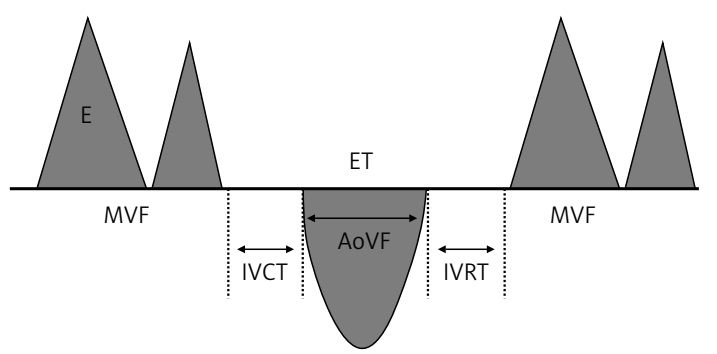

Tei Index $=\frac{\text { IVCT }+ \text { IVRT }}{E T}$

Figure 2. Method of Tei index measurements

AoVF - aortic valve flow, IVCT - isovolumetric contraction time, IVRT - isovolumetric relaxation time, ET - ejection time, MVF- mitral valve inflow

\section{Evaluation of right ventricular diastolic function}

Inflow to the RV through the tricuspid valve was recorded in the apical four-chamber view with the Doppler gate positioned in the RV at the level of the tricuspid valve leaflet edges. Peak velocity of the early inflow phase $(E)$, peak velocity of the atrial inflow phase $(A)$ and $E / A$ ratio were evaluated.

\section{Tissue Doppler mitral annular early} diastolic velocities

Tricuspid annulus early diastolic velocity (TR E' lateral) was measured. Tricuspid E/E' was also calculated. Tricuspid E/A $<0.8$ and E/E' $>6$ were defined as abnormal [19].

\section{Assessment of global right ventricular performance}

The index of RV efficiency (Tei index) was calculated according to the method proposed by Tei et al. A normal value for the RV was assumed to be $0.28 \pm 0.04$ [23].

\section{Statistical analysis}

Data characterized by a normal distribution are expressed as a mean followed by a stan- dard deviation. For comparison of two groups for the quantitative parameters Wilcoxon test for independent samples (Wilcoxon rank-sum test) was used. To analyze changes in quantitative parameters during follow-up Wilcoxon test for related samples (Wilcoxon signed-rank test) was performed. For categorical variables the differences between the groups were compared with the $\chi^{2}$ test or Fisher's exact test. Correlations between echocardiographic parameters and TIMP-1 were evaluated by Spearman's correlation coefficients.

An analysis was performed using a statistical software package (SAS 9.2). Value of $p<0.05$ was considered statistically significant.

\section{Results}

A total of 111 SSc patients and 21 control subjects were enrolled in this study. The general characteristics of the study population and control group are summarized in Table I.

\section{Treatment}

Angiotensin-converting enzyme inhibitors (ACE-I) were taken by 31 (28\%) SSc patients, angiotensin II receptor antagonists by $7(6 \%)$, $\beta$-adrenolytics by 10 (9\%), diuretics by 16 (14\%), calcium channel blockers by 27 (24\%). Due to the progression of SSc 15 (13.5\%) patients received immunosuppressant agents (glucocorticoids and cyclophosphamide). We did not find statistically significant differences in the use of cardiovascular drugs between the SSc and control group.

The main clinical, pulmonary function and serological findings of SSc patients are shown in Table II. Echocardiographic parameters of LV diastolic function in SSc patients and controls are outlined in Table III.

The mean LA diameter in parasternal long axis view did not differ significantly between the groups (32.8 $\pm 4.73 \mathrm{~mm}$ vs. $31.8 \pm 3.47 \mathrm{~mm}, \mathrm{NS})$. Moreover, the mean upper to lower and medial to lateral dimensions of the LA were also similar in

Table I. General parameters in the SSc and the control group

\begin{tabular}{|lccc|}
\hline Parameter & SSc patients $(n=111)$ & Control subjects $(n=21)$ & Value of $p$ \\
\hline Age [years] & $54.2 \pm 13.7$ & $49.3 \pm 10.5$ & NS \\
\hline Gender (F/M), no. & $101 / 10$ & $18 / 3$ & NS \\
\hline Body surface area [m $\left.{ }^{2}\right]$ & $1.73 \pm 0.26$ & $121 \pm 12$ & NS \\
\hline Systolic blood pressure [mm Hg] & $124 \pm 21$ & $72 \pm 9$ & NS \\
\hline Diastolic blood pressure [mm Hg] & $76 \pm 11$ & $76.21 \pm 12.38$ & NS \\
\hline Heart rate [bpm] & $73.47 \pm 9.45$ & $6(29 \%)$ & NS \\
\hline Systemic hypertension [\%] & $37(33 \%)$ & & \\
\hline
\end{tabular}


Table II. Clinical, pulmonary function and serological data of 111 SSc patients

\begin{tabular}{|c|c|c|}
\hline Characteristics & Mean & \\
\hline Disease duration [years] & $9.0 \pm 12.4$ (range: $1-25)$ & \\
\hline FVC, \% predicted & $100.4 \pm 18.3$ & \\
\hline $\mathrm{FEV}_{1}, \%$ predicted & $94.7 \pm 19.7$ & \\
\hline $\mathrm{FEV}_{1} / \mathrm{FVC}, \%$ predicted & $79.8 \pm 8.2$ & \\
\hline TLC, \% predicted $(n=24)$ & $101.9 \pm 17.5$ & \\
\hline DLCO, \% predicted $(n=21)$ & $70.7 \pm 19.1$ & \\
\hline Parameter & Number & Percent \\
\hline \multicolumn{3}{|l|}{ Cutaneous involvement: } \\
\hline Diffuse & 47 & 42 \\
\hline Limited & 64 & 58 \\
\hline \multicolumn{3}{|l|}{ Autoantibodies: } \\
\hline ANA positive & 105 & 95 \\
\hline ACA positive & 34 & 31 \\
\hline Anti-Scl 70 positive & 56 & 50 \\
\hline
\end{tabular}

$F V C$ - forced vital capacity, FEV - forced expiratory volume in $1 \mathrm{~s}, T L C$ - total lung capacity, DLCO - diffusion capacity of the lung for carbon monoxide

Table III. Echocardiographic evaluation of left ventricular diastolic performance in SSc patients and controls

\begin{tabular}{|lccc|}
\hline Parameter & SSc $(n=111)$ & Control group $(n=21)$ & Value of $p$ \\
\hline Mit E [cm/s] & $73.24 \pm 17.04$ & $87.4 \pm 14.25$ & NS \\
\hline Mit A [cm/s] & $76.92 \pm 18.55$ & $66.45 \pm 16.15$ & 0.01 \\
\hline Mit E/A & $0.98 \pm 0.3$ & $1.21 \pm 0.28$ & 0.002 \\
\hline IVRT [ms] & $73.25 \pm 12.0$ & $64.28 \pm 7.79$ & 0.001 \\
\hline DT [ms] & $178.95 \pm 38.84$ & $185.6 \pm 25.63$ & NS \\
\hline PVF S [cm/s] & $61.14 \pm 17.24$ & $60.39 \pm 10.85$ & NS \\
\hline PVF D [cm/s] & $47.22 \pm 11.74$ & $50.65 \pm 9.47$ & NS \\
\hline S/D & $1.32 \pm 0.4$ & $1.21 \pm 0.28$ & NS \\
\hline PVF Ar [cm/s] & $29.99 \pm 7.22$ & $29.47 \pm 3.62$ & NS \\
\hline LV Tei index & $0.46 \pm 0.09$ & $0.39 \pm 0.06$ & 0.01 \\
\hline Mit E' lateral [cm/s] & $10.87 \pm 3.82$ & $12.51 \pm 4.25$ & NS \\
\hline Mit E/E' lateral & $7.55 \pm 2.85$ & $6.87 \pm 2.30$ & NS \\
\hline Mit E' septal [cm/s] & $8.12 \pm 2.35$ & $10.1 \pm 3.61$ & 0.02 \\
\hline Mit E/E' septal & $9.69 \pm 3.05$ & $8.46 \pm 2.59$ & NS \\
\hline
\end{tabular}

SSc patients and controls ( $36.0 \pm 4.0$ vs. $35.5 \pm 3.3$, NS and $44.7 \pm 6.2$ vs. $45.1 \pm 4.1$, NS).

Impaired LV relaxation defined as E/A ratio of the mitral inflow < 0.8 was observed in 38 (34\%) patients with SSc and in 1 (5\%) of the control group ( $p<0.001)$. The mean $\mathrm{E} / \mathrm{A}$ ratio was significantly lower in patients with SSc. No marked differences between the two groups in pulmonary vein inflow parameters were observed. However, significantly higher mean value of the Tei index was seen in SSc patients compared to controls. We found no significant differences in mitral DTI parameters except for Mit E' septal, whose mean value was lower in SSc patients than controls.

In SSc patients the mean value of ejection fraction (EF) was significantly lower than in the control group ( $65.05 \pm 5.1 \%$ vs. $67.57 \pm 2.52 \%, p=0.01$ ). 
The echocardiographic parameters of RV diastolic function are presented in Table IV. No significant differences were found in the mean upper to lower and medial to lateral dimensions of the RA in SSc patients and controls (42.8 $\pm 5.2 \mathrm{~mm}$ vs. $40.1 \pm 4.8 \mathrm{~mm}$, NS and $35.4 \pm 4.6$ vs. $3.2 \pm 3.7$; NS, respectively). Tricuspid $\mathrm{E} / \mathrm{A}<0.8$ was detected in $25(22.5 \%)$ SSc patients and in 1 (5\%) control subject $(p<0.001)$.

The SSc patients had a significantly higher mean value of the Tei index and lower E/A ratio than control individuals.

The mean value of FAC did not differ between SSc patients and controls ( $43.13 \pm 9.15 \%$ vs. 40.13 $\pm 9.48 \%$, NS), while the mean value of TAPSE was significantly lower in SSc patients $(22.2 \pm 3.23$ vs. $24.14 \pm 2.37, p=0.01$ ).

In the study group the mean value of TRPG was significantly higher than in controls (26.67 $\pm 6.97 \mathrm{~mm} \mathrm{Hg}$ vs. $17.78 \pm 4.07 \mathrm{~mm} \mathrm{Hg}, p<0.0001$ ). Twenty-eight patients (25\%) with TRPG exceeding
$31 \mathrm{~mm} \mathrm{Hg}$ were referred for RHC. Seven refused consent to undergo the procedure, 1 patient could not undergo the procedure due to rapid deterioration of general condition, and 1 patient died while awaiting the procedure due to bleeding from esophageal varices. Finally, RHC was performed in 19 patients. In 2 patients pulmonary arterial hypertension and in 1 pulmonary venous hypertension was diagnosed. We did not find any differences in $\mathrm{LV}$ and RV diastolic parameters between patients with TRPG $\geq 31 \mathrm{~mm} \mathrm{Hg}$ and below this value.

After at least 1 year of observation $(3.0 \pm 1.1$ years, median 3.1 years, range $1.0-4.8$ years) the second evaluation of 69 SSC patients was done. After the follow-up period the incidence of ACE I treatment was significantly higher than at the baseline (31 (45\%) vs. 31 (28\%), $p<0.001)$. We did not find statistically significant differences in the use of other drugs between baseline and follow-up.

The LV diastolic parameters at baseline and after follow-up are summarized in Table V. The mean

Table IV. Echocardiographic evaluation of right ventricular diastolic function

\begin{tabular}{|lccc|}
\hline Parameter & SSc $(n=111)$ & Control group $(n=21)$ & Value of $p$ \\
\hline $\operatorname{Tr}$ E $[\mathrm{cm} / \mathrm{s}]$ & $52.12 \pm 11.35$ & $58.85 \pm 11.15$ & 0.008 \\
\hline $\operatorname{Tr}$ A [cm/s] & $50.49 \pm 13.53$ & $46.43 \pm 10.38$ & NS \\
\hline $\operatorname{Tr}$ E/A & $1.05 \pm 0.24$ & $1.3 \pm 0.27$ & 0.0003 \\
\hline RV Tei index & $0.37 \pm 0.08$ & $0.29 \pm 0.02$ & $<0.0001$ \\
\hline $\operatorname{Tr} E^{\prime}[\mathrm{cm} / \mathrm{s}]$ & $12.04 \pm 3.55$ & $12.66 \pm 2.68$ & NS \\
\hline $\operatorname{Tr}$ E/E' & $4.75 \pm 1.84$ & $4.67 \pm 0.82$ & NS \\
\hline
\end{tabular}

Table V. LV diastolic parameters at baseline examination and after follow-up

\begin{tabular}{|lccc|}
\hline Parameter & SSc $(n=69)$ & SSc follow-up $(n=69)$ & Value of $p$ \\
\hline Mit E & $71.8 \pm 14.95$ & $71.5 \pm 17.1$ & NS \\
\hline Mit A & $75.5 \pm 15.5$ & $79.23 \pm 19.9$ & NS \\
\hline Mitral E/A & $0.99 \pm 0.3$ & $0.92 \pm 0.3$ & 0.02 \\
\hline IVRT [ms] & $73.45 \pm 11.13$ & $75.0 \pm 13.19$ & NS \\
\hline DT [ms] & $175.64 \pm 36.63$ & $198.66 \pm 36.63$ & 0.001 \\
\hline PVF S [cm/s] & $59.45 \pm 16.37$ & $63.57 \pm 16.62$ & 0.03 \\
\hline PVF D [cm/s] & $46.72 \pm 11.56$ & $47.11 \pm 12.18$ & NS \\
\hline S/D & $1.21 \pm 0.28$ & $1.38 \pm 0.33$ & 0.05 \\
\hline PVF Ar [cm/s] & $29.48 \pm 7.46$ & $33.28 \pm 8.06$ & 0.0003 \\
\hline LV Tei index & $0.45 \pm 0.1$ & $0.47 \pm 0.1$ & NS \\
\hline Mit E' lateral [cm/s] & $10.87 \pm 3.8$ & $11.0 \pm 3.1$ & NS \\
\hline Mit E/E' lateral & $7.5 \pm 3.11$ & $6.91 \pm 2.53$ & NS \\
\hline Mit E' septal [cm/s] & $8.1 \pm 2.3$ & $7.9 \pm 2.3$ & NS \\
\hline Mit E/E' septal & $9.47 \pm 3.3$ & $9.56 \pm 3.47$ & NS \\
\hline
\end{tabular}


Table VI. RV diastolic parameters at baseline and after follow-up period

\begin{tabular}{|lccc|}
\hline Parameter & SSc $(n=69)$ & SSc follow-up $(n=69)$ & Value of $p$ \\
\hline $\operatorname{Tr} E$ & $53.01 \pm 10.6$ & $50.9 \pm 10.6$ & NS \\
\hline $\operatorname{Tr} A$ & $51.1 \pm 13.9$ & $50.5 \pm 11.3$ & NS \\
\hline Tricuspid E/A & $1.05 \pm 0.22$ & $1.02 \pm 0.24$ & NS \\
\hline RV Tei index & $0.35 \pm 0.07$ & $0.4 \pm 0.08$ & NS \\
\hline $\operatorname{Tr}$ E' $[\mathrm{cm} / \mathrm{s}]$ & $12.1 \pm 3.5$ & $11.6 \pm 3.0$ & NS \\
\hline Tricuspid E/E' & $4.75 \pm 1.35$ & $4.83 \pm 1.54$ & \\
\hline
\end{tabular}

value of mitral E/A was significantly lower after follow-up in SSc patients. Mean DT was significantly longer than in baseline examination. Moreover, the mean PVF S velocity and the mean PVF Ar velocity were higher and the mean S/D was marginally higher after follow-up. We did not find any significant differences in DTI parameters between baseline and follow-up. In SSc patients the mean value of EF decreased significantly after follow-up $(66.54 \pm 3.03$ vs. $65.1 \pm 4.18, p=0.03)$.

The RV diastolic parameters at baseline and after follow-up are presented in Table VI. Among the parameters of RV diastolic functionality the mean value of RV Tei index was significantly higher after the follow-up period than at baseline examination. We did not find any significant differences in the mean value of FAC and TAPSE between baseline and follow-up (43.13 $\pm 9.15 \%$ vs. $40.99 \pm 13.15 \%$, NS and $22.77 \pm 2.32$ vs. $21.41 \pm 2.95$, NS, respectively). The mean value of TRPG was significantly increased after observation $(26.0 \pm 7.18 \mathrm{~mm} \mathrm{Hg}$ vs. $27.09 \pm 7.28 \mathrm{~mm} \mathrm{Hg}, p=0.01$ ).

\section{Tissue inhibitors of metalloproteinase 1 assessment}

At baseline the mean serum level of TIMP-1 did not differ between SSc patients and controls (167.0 \pm 63 vs. $182.8 \pm 29 \mathrm{ng} / \mathrm{ml}, \mathrm{NS}$ ); however, it was significantly elevated in the follow-up group compared to baseline examination $(161.6 \pm 59 \mathrm{ng} / \mathrm{ml}$ vs. $204.6 \pm 63 \mathrm{ng} / \mathrm{ml}, p=0.0001$ )

Significant correlations between TIMP-1 serum level and DTI parameters in SSc follow-up patients are presented in Table VII.

The mean serum TIMP-1 level correlated negatively with Mit E lateral and septal and positively with E/E', both septal and lateral.

\section{Discussion}

Diastolic dysfunction is clinically important and there is growing evidence on LV relaxation impairment in SSc patients. However, RV diastolic function has not been widely studied yet. In one of the largest studies on LV diastolic function, De Groote et al. revealed LV diastolic dysfunction on
Table VII. Significant correlations between TIMP-1 serum level and DTI parameters

\begin{tabular}{|lcc|}
\hline Parameter & $r$ & $p$ \\
\hline Mit E lateral & -0.46 & 0.0003 \\
\hline Mit E septal & -0.38 & 0.003 \\
\hline Mit E/E' lateral & 0.4 & 0.002 \\
\hline Mit E/E' septal & 0.32 & 0.01 \\
\hline
\end{tabular}

echocardiographic examination in 101 (17.7\%) of 570 SSc patients [10]. Meune et al. reported, using DTI parameters, diastolic dysfunction in 30 (30\%) SSc patients with a group of 100 examined individuals [1]. In an interesting paper, Hachulla et al. analyzed magnetic resonance imaging in $52 \mathrm{SSc}$ patients and detected impaired diastolic function in 15 (35\%) of 45 patients [24]. Our results are consistent with data from the literature. We revealed impaired relaxation of LV (E/A < 0.8$)$ in 38 (34\%) patients with SSc and in only one subject (5\%) from the control group $(p<0.001)$. The PVF parameters did not differ between groups, but there was an evident trend to their inferior value in SSc patients compared to control subjects. The LV Tei index combines both elements of systolic and diastolic LV function evaluation. It has been shown that heart rate, blood pressure and afterload do not affect the LV Tei index [22, 25]. However, it is partial preload dependent and less reliable when LA or RA pressure is elevated [23]. The Tei index value is increased in patients with LV diastolic dysfunction and shows a correlation with hemodynamic parameters describing LV relaxation [26, 27]. In our group of SSc patients we observed a significantly increased mean value of LV Tei index compared to controls $(p=0.01)$. However, it should be underlined that although left ventricular systolic function was preserved in SSC patients, it was slightly impaired in comparison to controls. The advantage of our study was to assess diastolic function after follow-up. Few data have been published on this issue so far. Moreover, we performed detailed echo evaluation of both ventricular functions using important echo 
parameters including DTI and PVF. In SSc patients only Maione et al. analyzed LV diastolic function after the follow-up period. However, this was an evaluation without DTI and PVF parameters assessment. After a mean time of observation of 65 \pm 36 months they revealed significantly decreased Mit E/A (1.08 \pm 0.37 vs. $1.2 \pm 0.49, p<0.001)$. However, DT and IVRT did not differ significantly between baseline and follow-up examinations [15]. Our results suggest ongoing deterioration of the LV diastolic function after follow-up. The mean value of E/A decreased (0.92 \pm 0.3 vs. $0.99 \pm 0.3$, $p=0.02$ ) and the mean value of DT significantly increased after observation. Moreover, in our group of patients the mean values of PVF parameters deteriorated after follow-up, suggesting impaired LV relaxation. The RV diastolic function assessment is much more difficult mainly due to the methodological limitations; however, its measurements should be considered in patients with suspected RV impairment as a marker of early or subtle RV dysfunction [19]. There are only a few data about RV diastolic function in SSc patients. Giunta et al. reported RV diastolic dysfunction in 31 (40\%) of 77 SSc patients and in none of the control group [11]. Similar findings were reported by Lindqvist et al. They revealed impaired RV diastolic function in a small group of $26 \mathrm{SSc}$ patients compared to controls (Tr E/A $1.2 \pm 0.4$ vs. $1.7 \pm 0.6, p<0.01$ ) [28]. In our group of SSc patients we revealed impaired RV relaxation. According to the recent guidelines for echocardiographic assessment of the right heart in adults, tricuspid E/A ratio $<0.8$ is considered as abnormal [19]. E/A $<0.8$ was detected in 25 (22.5\%) SSc patients and in 1 (5\%) control subject ( $p<0.001)$. The mean value of tricuspid E/A was significantly lower in the study group $(p=0.0003)$. Moreover, the mean value of the RV Tei index, a parameter of global estimation of both systolic and diastolic function of RV, was significantly increased in SSc patients $(p<0.0001)$. There are some data about the prognostic value of RV Tei index in patients with pulmonary hypertension [23]. Furthermore, changes of the RV Tei index correlate with clinical status in this group of patients [29]. So far there is limited evidence about the RV Tei index in SSc patients. Recently, Ivanovic et al. reported an increased RV Tei index in 50 SSc patients compared to controls $(p<0.001)$ [30].

After the follow-up period the mean RV Tei index value increased ( $p=0.01)$, although other parameters of RV diastolic assessment (tricuspid E/A and DTI indices) did not differ between baseline and follow-up examination. Our observations suggest that RV diastolic function is impaired in comparison to control subjects but did not get significantly worse after follow-up. According to our knowledge, in the available literature there are no data on long-term observation of RV diastolic function in SSc patients.

In our opinion observed differences between LV and RV diastolic parameters in SSc patients and controls could not be explained by coexisting systemic hypertension because we did not find differences in prevalence of this condition between groups. Moreover, patients with essential systemic hypertension and LV hypertrophy were excluded.

It is well known that ACE I can improve diastolic function in hypertensive patients [31]. What is interesting, the differences in LV diastolic parameters in SSc patients were also evident after the follow-up period despite more frequent use of ACE I compared to baseline.

Many studies have confirmed raised serum level of TIMP-1 in SSc patients [3, 32]. Lindsay et al. analyzed echocardiographic data of 100 hypertensive patients, and reported that TIMP-1 is a marker of LV diastolic dysfunction. Moreover, there are several studies where the correlation between serum TIMP-1 level and arterial stiffness was confirmed [33, 34]. In our SSc follow-up group the mean serum concentration of TIMP-1 was significantly elevated when compared to baseline examination. What is interesting, after the follow-up period significant correlations between the mean value of serum TIMP-1 concentrations and DTI parameters were evident. Probably the progression of heart fibrosis and arterial stiffness during the observation, expressed by progressive diastolic dysfunction and increased serum concentrations of TIMP-1, led to such a correlation. In our opinion this is a new, original observation. In only one study involving 42 patients with SSc, Shahin et al. observed frequent diastolic dysfunction in patients with elevated levels of TIMP-2 [35].

Other authors have tried to find other biomarkers that correlate with diastolic dysfunction. Recently, Jurisic et al. reported that IL-6 levels are increased in patients with SSc and correlate with LV diastolic dysfunction [36]. More studies are needed investigating the relationship of other biomarkers with diastolic dysfunction in patients with SSc.

In this study we did not analyze the potential impact of lung function parameters on the echocardiographic indices. However, in order to minimize the influence of impaired lung function, patients with even moderate airway obstruction were not included in the study.

In our group of patients we did not assess other biomarkers of matrix remodeling. Perhaps their assessment could provide a better insight into the relationship between matrix remodeling and diastolic function in SSc patients.

In conclusion, left and right ventricular relaxation is impaired in SSc patients when compared to controls. Moreover, left ventricular diastolic 
function deteriorated after the follow-up period. The TIMP-1 serum levels correlate with echocardiographic parameters, providing a potent link for LV diastolic function and matrix remodeling in patients with SSc.

\section{References}

1. Meune C, Avouac J, Wahbi K, et al. Cardiac involvement in systemic sclerosis assessed by tissue-doppler echocardiography during routine care: A controlled study of 100 consecutive patients. Arthritis Rheum 2008; 58: 1803-9.

2. Dziankowska-Bartkowiak B, Gerlicz-Kowalczuk Z, Waszczykowska E. Angiogenin and SDF-1alpha serum concentration in patients with systemic sclerosis in relation to clinical status. Arch Med Sci 2011; 7: 92-6.

3. Blaise S, Maas R, Trocme C, et al. Correlation of biomarkers of endothelium dysfunction and matrix remodeling in patients with systemic sclerosis. J Rheumatol 2009; 36: 984-8.

4. Toubi E, Kessel A, Grushko G, Sabo E, Rozenbaum M, Rosner I. The association of serum matrix metalloproteinases and their tissue inhibitor levels with scleroderma disease severity. Clin Exp Rheumatol 2002; 20: 221-4.

5. Young-Min SA, Beeton C, Laughton R, et al. Serum TIMP-1, TIMP-2, and MMP-1 in patients with systemic sclerosis, primary Raynaud's phenomenon, and in normal controls. Ann Rheum Dis 2001; 60: 846-51.

6. Kikuchi K, Kubo M, Sato S, Fujimoto M, Tamaki K. Serum tissue inhibitor of metalloproteinases in patients with systemic sclerosis. J Am Acad Dermatol 1995; 33: 973-8.

7. Kim WU, Min SY, Cho ML, et al. Elevated matrix metalloproteinase- 9 in patients with systemic sclerosis. Arthritis Res Ther 2005; 7: R71-9.

8. Young-Min SA, Beeton C, Laughton R, et al. Serum TIMP-1, TIMP-2, and MMP-1 in patients with systemic sclerosis, primary Raynaud's phenomenon, and in normal controls. Ann Rheum Dis 2001; 60: 846-51.

9. Hinchcliff M, Desai CS, Varga J, Shah SJ. Prevalence, prognosis, and factors associated with left ventricular diastolic dysfunction in systemic sclerosis. Clin Exp Rheumatol 2012; 30 (2 Suppl 71): S30-7.

10. de Groote P, Gressin V, Hachulla E, et al. Evaluation of cardiac abnormalities by Doppler echocardiography in a large nationwide multicentric cohort of patients with systemic sclerosis. Ann Rheum Dis 2008; 67: 31-6.

11. Giunta A, Tirri E, Maione S, et al. Right ventricular diastolic abnormalities in systemic sclerosis. Relation to left ventricular involvement and pulmonary hypertension. Ann Rheum Dis 2000; 59: 94-8.

12. Kazzam E, Waldenstrom A, Landelius J, Hallgren R, Arvidsson A, Caidahl K. Non-invasive assessment of left ventricular diastolic function in patients with systemic sclerosis. J Intern Med 1990; 228: 183-92.

13. Maione S, Valentini G, Giunta A, et al. Evaluation of cardiac structures and function in systemic sclerosis by Doppler echocardiography. Cardiology 1991; 79: 165-71.

14. Poanta L, Dadu R, Tiboc C, Rednic S, Dumitrascu D. Systolic and diastolic function in patients with systemic sclerosis. Eur J Intern Med 2009; 20: 378-82.

15. Maione S, Cuomo G, Giunta A, et al. Echocardiographic alterations in systemic sclerosis: a longitudinal study. Semin Arthritis Rheum 2005; 34: 721-7.

16. Preliminary criteria for the classification of systemic sclerosis (scleroderma). Subcommittee for scleroderma criteria of the American Rheumatism Association Diagnostic and Therapeutic Criteria Committee. Arthritis Rheum 1980; 23: 581-90.

17. Valentini G, Bencivelli W, Bombardieri S, et al. European Scleroderma Study Group to define disease activity criteria for systemic sclerosis. III. Assessment of the construct validity of the preliminary activity criteria. Ann Rheum Dis 2003; 62: 901-3.

18. Feigenbaum H, Armstrong WF, Ryan T. Feigenbaum's echocardiography. 6 ed. Lippincott, Williams \& Wilkins, Philadelphia 2005.

19. Rudski LG, Lai WW, Afilalo J, et al. Guidelines for the echocardiographic assessment of the right heart in adults: a report from the American Society of Echocardiography endorsed by the European Association of Echocardiography, a registered branch of the European Society of Cardiology, and the Canadian Society of Echocardiography. J Am Soc Echocardiogr 2010; 23: 685-713.

20. Galie N, Hoeper MM, Humbert M, et al. Guidelines for the diagnosis and treatment of pulmonary hypertension: the Task Force for the Diagnosis and Treatment of Pulmonary Hypertension of the European Society of Cardiology (ESC) and the European Respiratory Society (ERS), endorsed by the International Society of Heart and Lung Transplantation (ISHLT). Eur Heart J 2009; 30: 2493-537.

21. Nagueh SF, Appleton CP, Gillebert TC, et al. Recommendations for the evaluation of left ventricular diastolic function by echocardiography. Eur J Echocardiogr 2009; 10: 165-93.

22. Tei C, Ling LH, Hodge DO, et al. New index of combined systolic and diastolic myocardial performance: a simple and reproducible measure of cardiac function - a study in normals and dilated cardiomyopathy. J Cardiol 1995; 26: 357-66.

23. Tei C, Dujardin KS, Hodge DO, et al. Doppler echocardiographic index for assessment of global right ventricular function. J Am Soc Echocardiogr 1996; 9: 838-47.

24. Hachulla AL, Launay D, Gaxotte V, et al. Cardiac magnetic resonance imaging in systemic sclerosis: a cross-sectional observational study of 52 patients. Ann Rheum Dis 2009; 68: 1878-84.

25. Karatzis EN, Giannakopoulou AT, Papadakis JE, Karazachos AV, Nearchou NS. Myocardial performance index (Tei index): evaluating its application to myocardial infarction. Hellenic J Cardiol 2009; 50: 60-5.

26. Lacorte JC, Cabreriza SE, Rabkin DG, et al. Correlation of the Tei index with invasive measurements of ventricular function in a porcine model. J Am Soc Echocardiogr 2003; 16: 442-7.

27. Tei C, Nishimura RA, Seward JB, Tajik AJ. Noninvasive Doppler-derived myocardial performance index: correlation with simultaneous measurements of cardiac catheterization measurements. J Am Soc Echocardiogr 1997; 10: 169-78.

28. Lindqvist P, Caidahl K, Neuman-Andersen G, et al. Disturbed right ventricular diastolic function in patients with systemic sclerosis: a Doppler tissue imaging study. Chest 2005; 128: 755-63.

29. Sebbag I, Rudski LG, Therrien J, Hirsch A, Langleben D. Effect of chronic infusion of epoprostenol on echocardiographic right ventricular myocardial performance index and its relation to clinical outcome in patients with primary pulmonary hypertension. Am J Cardiol 2001; 88: 1060-3.

30. Ivanovic BA, Tadic MV, Zlatanovic MM, Damjanov NN, Ostojic PM, Bonaci-Nikolic BN. Which factors impact 
myocardial function in systemic sclerosis? Echocardiog raphy 2012; 29: 307-17.

31. Terpstra WF, May JF, Smit AJ, et al. Long-term effects of amlodipine and lisinopril on left ventricular mass and diastolic function in elderly, previously untreated hypertensive patients: the ELVERA trial. J Hypertens 2001; 19: 303-9.

32. Young-Min SA, Beeton C, Laughton R, et al. Serum TIMP-1, TIMP-2, and MMP-1 in patients with systemic sclerosis, primary Raynaud's phenomenon, and in normal controls. Ann Rheum Dis 2001; 60: 846-51.

33. Stakos DA, Tziakas DN, Chalikias GK, Mitrousi K, Tsigalou C, Boudoulas H. Associations between collagen synthesis and degradation and aortic function in arterial hypertension. Am J Hypertens 2010; 23: 488-94.

34. Zureik M, Beaudeux JL, Courbon D, Benetos A, Ducimetiere P. Serum tissue inhibitors of metalloproteinases 1 (TIMP-1) and carotid atherosclerosis and aortic arterial stiffness. J Hypertens 2005; 23: 2263-8.

35. Shahin A, Elsawaf A, Ramadan S, Shaker O, Amin M Taha M. Serum levels of tissue inhibitors of metalloproteinase 2 in patients with systemic sclerosis with duration more than 2 years: correlation with cardiac and pulmonary abnormalities. Mediators Inflamm 2006; 2006: 38458.

36. Jurisic Z, Martinovic-Kaliterna D, Marasovic-Krstulovic D, et al. Relationship between interleukin- 6 and cardiac in volvement in systemic sclerosis. Rheumatology (Oxford) 2013; 52: 1298-302 